\title{
Evaluación del sellado apical de Bioroot RCS, Tubliseal y Top Seal: estudio in vitro
}

\section{Bioroot RCS, Tubliseal and Top Seal apical sealing evaluation: in vitro study}

\author{
Fernanda Sacoto* ${ }^{*}$, Paula Cárcamo², Raúl Alcántara² \\ 1 Docente de la Universidad Católica de Cuenca. \\ 1 Estudiante de la carrera de odontología de la Universidad Católica de Cuenca. \\ *kfer18_fisha@hotmail.es
}

DOI: https://

\section{Resumen}

En endodoncia, la fase de obturación radicular tiene como objetivo el sellado hermético del sistema de conductos radiculares, para conseguir esto se utilizan diversos materiales de obturación y selladores. Los selladores biocerámicos han estado disponible para su uso en endodoncia durante los últimos 30 años, tomando relevancia actualmente con el aumento del uso de la tecnología en el campo biocerámico. Este sellador ha sido ampliamente investigado por sus propiedades biocompatibles, registrándose escasa información sobre su capacidad de sellado apical. Por esta razón nuestro estudio evaluó esta característica midiendo el grado de filtración apical utilizando azul de metileno. Para ello se seleccionaron 80 dientes humanos extraídos con conducto único, se decoronaron y los conductos fueron preparados quimiomecanicamente con el sistema rotatorio Protaper Next hasta calibre X3. Previo a la obturación las muestras se dividieron en 4 grupos aleatoriamente: 1) Tubliseal, 2) Topseal, 3) BioRoot RCS y 4 ) Control positivo sin sellador. Se procedió a obturar con la técnica de cono único y los selladores respectivos excepto el grupo control. Las muestras se sumergieron en una solución de azul de metileno por 24 horas, se lavaron en agua corriente y se realizó la diafanización. Las muestras diafanizadas se observaron con estereoscopio y se midió la penetración lineal del colorante en micrones. 
Para el análisis de resultados se utilizó la prueba estadística Kruskal Wallis, mostrando que existe una diferencia significativa de los grupos experimentales con el grupo de control. A pesar, que no se presenta una diferencia significativa entre los grupos experimentales, Tubliseal tiene el mejor comportamiento como sellador apical. Los selladores BioRoot RCS y Topseal tuvieron comportamientos similares. Concluimos que se deben ajustar algunos parámetros metodológicos con el fin de obtener resultados más exactos en investigaciones futuras.

Palabras clave: Sellado apical, filtración apical, Tubliseal, Topseal, Bioroot RCS.

\section{Abstract}

In endodontics, the root filling phase aims to hermetically seal the root canal system. To achieve this, various sealing materials and sealants are used. Bioceramic sealants have been available for endodontics use during the past 30 years. Nowadays, with the increasing use of technology in the bioceramic field, these sealants have gain relevance and have been extensively investigated because of their biocompatible characteristics. However, little information is provided on their apical sealing capacity. This is why our study evaluated this characteristic by measuring the apical filtration level using methylene blue. To achieve this, 80 human teeth extracted with a single conduit were selected, their crowns were taken out, and the conduits were chemically prepared with the Protaper Next rotary system up to X3 calibre. Before the fiIling, the samples were randomly divided into 4 groups: 1) Tubliseal, 2) Topseal, 3) BioRoot RCS and 4) Positive control without sealant. The obturation was performed using the single cone technique and the corresponding sealants, except the control group. The samples were then immersed in a methylene blue solution for 24 hours, washed in fresh water and diaphanized. The diaphanized samples were observed by stereoscopy, and the dye linear penetration was measured in microns.

The Kruskal Wallis statistical test was used for the analysis of the results, revealing a significant difference between the experimental groups and the control group. Despite there being no significant difference between the experimental groups, Tubliseal performed the best as an apical sealer. BioRoot RCS and Topseal sealants had similar performance. We conclude that some methodological parameters must be adapted to obtain more accurate results in future research.

Key words: Apical sealing, apical filtration, Tubliseal, Topseal, Bioroot RCS. 


\section{Introducción}

En la terapia endodóntica, la fase de obturación está relacionada en gran medida con el éxito y fracaso del tratamiento; el objetivo de la obturación es lograr un sellado hermético del sistema de conductos radiculares. Actualmente continúa la investigación de nuevos materiales con mejores propiedades para optimizar la calidad de la obturación, razón por la cual los materiales son sometidos a diversas pruebas que garanticen la obtención de resultados favorables en su aplicación clínica.

Estudios clínicos han revelado que la filtración apical de materiales de relleno radicular es una de las principales causas de los fracasos de la terapia endodóntica'

La obturación endodóntica limita el intercambio de fluidos entre el conducto y el área peri radicular, este fenómeno se denomina filtración y determina en gran parte el éxito del tratamiento. La importancia del sellado apical se explica en función de la filtración, de manera que si el material de obturación se solubiliza en el área apical, se pierde este sellado, permitiendo la filtración, y disminuyendo el éxito del tratamiento endodóntico. ${ }^{2}$

En el mercado tenemos nuevos materiales de obturación, dentro de los cuales se encuentran los selladores biocerámicos, este sellador ha sido ampliamente investigado por sus propiedades biocompatibles, registrando escasa información sobre su capacidad de sellado apical, lo que motiva a la realización de esta investigación.

El objetivo de este estudio es evaluar la capacidad de sellado apical de cementos de conducto: Tubliseal, Topseal y BioRoot RCS al ser utilizados en la obturación radicular de piezas uniradiculares humanas extraídas con técnica de cono único.

Los resultados que se obtengan de este estudio, proporcionarán información relevante para el clínico, al momento de la selección del tipo de sellador que otorgue un mejor sellado apical

\section{Marco Teórico}

La obturación del conducto radicular es un procedimiento en el cual el espacio del conducto radicular se rellena con materiales de llenado y sellado en la etapa final del tratamiento del conducto radicular, posterior a la limpieza y la conformación adecuada del mismo. ${ }^{3}$

La obturación radicular tiene como objetivo obtener un sellado tridimensional del sistema de conductos radiculares. Un relleno insuficiente puede traducirse en una reinfección del sistema de conductos radiculares, lo que irritaría el tejido periapical y comprometería el éxito del tratamiento. ${ }^{4}$

El sellado tridimensional hermético dificulta la comunicación del interior del conducto radicular con los tejidos periapicales. ${ }^{5}$ En la literatura se ha descrito que, la eficacia de sellado se ve afectada por la técnica de sellado, el tipo del sellador y los procedimientos clínicos realizados antes del llenado del conducto radicular. ${ }^{5}$ 
Según Cohen S et al, el sellado apical inadecuado es responsable de hasta el $60 \%$ de las fallas del tratamiento endodóntico. ${ }^{4}$ Por lo que es necesario que los materiales endodónticos proporcionen un sello a largo plazo y se evite filtraciones o fugas a nivel coronal y apical. ${ }^{6}$

En endodoncia, para lograr un sellado adecuado del conducto radicular, se están utilizando diversos materiales de obturación y selladores.

Los selladores se utilizan entre la superficie de la dentina y el núcleo obturador para llenar las oquedades que se crean, debido a la inhabilidad física del material que conforma el núcleo obturador para llenar todas las áreas del sistema de conductos.

Actualmente, en la obturación del conducto radicular se emplea una material de núcleo semisólido o sólido denominado gutapercha que va cementado en el conducto radicular con un sellador ${ }^{7}$, el cuál cumple funciones como sellar vacíos y canales accesorios ${ }^{8}$, formar una unión entre el núcleo del material de relleno y la pared del conducto ${ }^{9}$, actuar como lubricante para facilitar la colocación del núcleo de relleno y sepultar a las bacterias restantes. ${ }^{10}$

Un sellador ideal, según Grossman, debe cumplir con las siguientes propiedades ${ }^{10}$ :

- Ser pegajoso durante la mezcla para proporcionar buena adherencia a la pared del conducto una vez fraguado.

- Proporcionar un sellado hermético.

- Ser radiopaco para poder ser visualizado en las radiografías.

- Las partículas del polvo deben ser muy finas para que puedan ser mezcladas fácilmente con el líquido.

- No contraerse al fraguar.

- $\quad$ No teñir la estructura dental.

- Ser bacteriostático, o por lo menos no favorecer la proliferación bacteriana.

- Fraguar lentamente.

- $\quad$ Ser insoluble en fluidos tisulares.

- Ser tolerado por los tejidos, es decir, no producir irritación del tejido peri-radicular.

- Ser soluble en un solvente común, por si es necesario removerlo del conducto radicular.

Los selladores utilizados para la obturación radicular han sido elaborados en base a: óxido de zinc-eugenol, vidrio ionómero, resina, silicona, MTA, y biocerámicos. Los selladores de nueva generación están siendo diseñados para mejorar su capacidad de penetrar en los túbulos dentinarios y adherirse. 
Recientemente, se presentó en el mercado los selladores a base de silicato de calcio que recomiendan su uso con la técnica de obturación de cono único. Estos selladores también denominados biocerámicos son preconizados por sus propiedades favorables, sin embargo estos poseen un enfoque más biológico donde el sellador interactúa con la dentina." Estudios demuestran que la técnica de cono único disminuye el estrés en el diente generado por otras técnicas de obturación, obteniendo menos grietas apicales e incluso menor porosidad. ${ }^{3}$ A pesar de ello es importante entender que las propiedades físicas, químicas y biológicas son propias de cada sellador, por lo que esta técnica de cono único podría no ser la más indicada según el caso.

Los selladores basados en eugenol todavía se usan hoy en día debido a su larga historia. Si se preparan selladores a base de eugenol, el eugenol se mezcla con óxido de zinc en presencia de agua y se produce una reacción de quelación, que da como resultado una matriz de eugenolato de zinc con moléculas de eugenol sin reaccionar atrapadas en su interior. Debido a que es una reacción reversible, cuando el cemento fraguado entra en contacto con el agua, se produce la hidrólisis de eugenolato y libera eugenol. Además de esto, se liberan moléculas de eugenol atrapadas en la matriz. ${ }^{12}$

El sellador Topseal se presenta en dos componentes pasta/pasta basado en resinas epoxyaminas. Se ha atribuído características como el sellado de larga duración, buena estabilidad dimensional, propiedades auto-adhesivas y excelente radiopacidad. ${ }^{13}$

Los selladores en base a biocerámicos han estado disponibles para su uso en endodoncia durante los últimos treinta años. Este tipo de sellador tomó relevancia con el aumento del uso de la tecnología en el campo biocerámico. Estos materiales están diseñados específicamente para uso médico y odontológico. Están compuestos por alúmina, zirconio, vidrio bioactivo, cerámica de vidrio, hidroxiapatita y fosfato cálcico. ${ }^{10}$

Estos materiales son hidrofílicos, requieren agua para establecerse y poseen biocompatibilidad y la capacidad de formación de apatita ${ }_{1}^{14}$ la formación de tejido mineralizado se produce por el alto pH alcalino alcanzado que activa y estimula la expresión de fosfatasa alcalina ${ }^{8}$. El mecanismo exacto de interacción entre el sellador biocerámico y la dentina radicular es desconocido; sin embargo, se han sugerido los siguientes mecanismos para los selladores a base de silicato de calcio ${ }^{10}$ :

- $\quad$ "Difusión de las partículas de sellador en los túbulos dentinarios (difusión tubular) para producir enlaces mecánicos de enclavamiento,

- La infltración del contenido mineral del sellador en la dentina intertubular, que da como resultado el establecimiento de un mineral en la zona de filtración, produce una desnaturalización del tejido de colágeno con un sellador alcalino fuerte,

- Reacción parcial de fosfato con hidrogel de silicato de calcio e hidróxido de calcio, producido por la reacción de silicatos de calcio en presencia de la humedad de la dentina, que da como resultado la formación de hidroxiapatita a lo largo del mineral en la zona de filtración". 
Además de la biocompatibilidad presenta otras características como osteoconductividad, capacidad de lograr un sellado hermético excelente, formación de un enlace químico con la estructura dental, insolubilidad en los fluidos tisulares, buena radiopacidad y fácil manejo que han llevado al uso generalizado de los biocerámicos en el área endodóntica..$^{10}$

Las aplicaciones para su uso varían desde el recubrimiento pulpar directo, a la apexogénesis, la apexificación y reparación de la furca. ${ }^{15}$

Los biocerámicos se pueden clasificar como ${ }^{16-17-18}$ : bioinerte, bioactivo y biodegradable.

BioRoot RCS es un sellador biocerámico, basado en silicato tricálcico hidráulico, se presenta como polvo/líquido, elaborado por la compañía francesa Septodont y existente en el mercado desde el año 2015. ${ }^{14}$

Es preconizado por propiedades bioactivas, antimicrobianas y un óptimo sellado. En su composición posee una parte líquida que es una solución acuosa de cloruro de calcio y policarboxilato y la otra es un polvo que contiene silicato de tricalcio, povidona y óxido de zirconio, éste último le da la radiopacidad. ${ }^{14}$

Este sellador está basado en agua, por lo tanto se debe usar con una técnica de obturación de un solo cono en lugar de una compactación vertical caliente porque las propiedades de sellador se cambian cuando se calientan. ${ }^{11-19}$

Este sellador es libre de resina y eugenol, además presenta propiedades hidrofílicas y permite un aumento de los valores de Ph de más de $11 .^{8}$

Aunque las ventajas de estos materiales han contribuido a su rápida propagación en el campo dental, hoy en día, no son ampliamente utilizados, y los productos comercialmente disponibles en el mercado no son aún conocidos por muchos dentistas. ${ }^{2}$

\section{Metodología}

Se realizó un estudio experimental comparativo transversal que evaluó el sellado apical entre tres tipos de cementos selladores endodónticos, para lo que se utilizaron 80 dientes humanos extraídos con conducto radicular único, ápice formado y ausencia de caries radicular.

Las muestras fueron obtenidas por donación de odontólogos de atención primaria, los cuales firmaron un consentimiento informado en el que se indicó que las muestras tenían indicación de extracción con fines terapéuticos no asociadas al estudio.

Los criterios de exclusión considerados fueron: fractura radicular, dientes con más de un conducto, desarrollo radicular incompleto, rizálisis, presencia de caries radicular, conductos con calcificaciones, curvatura radicular severa según Schneider y tratamiento endodóntico previo. 
Los dientes unirradiculares fueron lavados y limpiados mediante destartraje manual con cureta de Gracey (Hu- Friedy) teniendo la precaución de no tocar el ápice de los dientes. Luego se sumergieron durante 2 horas en hipoclorito de sodio al 5,25\% para eliminar restos de tejidos orgánicos remanentes. Las coronas se seccionaron utilizando un disco de carburo (Dentorium) con motor de baja velocidad (NSK), cerca de la unión amelocementaria. Para determinar la longitud de trabajo, los conductos se permeabilizaron con lima K \#10 (Denstply, Maillefer) hasta ser visualizada en el foramen y se descontó $1 \mathrm{~mm}$.

Los conductos se limpiaron y conformaron con limas rotatorias Protaper Next (Dentsply, Maillefer) hasta X3 (Figura 1), manteniendo la permeabilidad con lima $\mathrm{K} \# 10$ y utilizando irrigación con hipoclorito de sodio a 2,5\% - 4ml/min- el que se removió con una cánula aspiradora endodóntica (Indusbello). Los conductos fueron sometidos a un protocolo de irrigación final con EDTA al 10\% (Hertz) por 1 minuto para remoción del barro destinario e irrigación con hipoclorito de sodio al 2,5\% - 1ml por conducto. Luego se secaron con conos de papel (Gapadent).

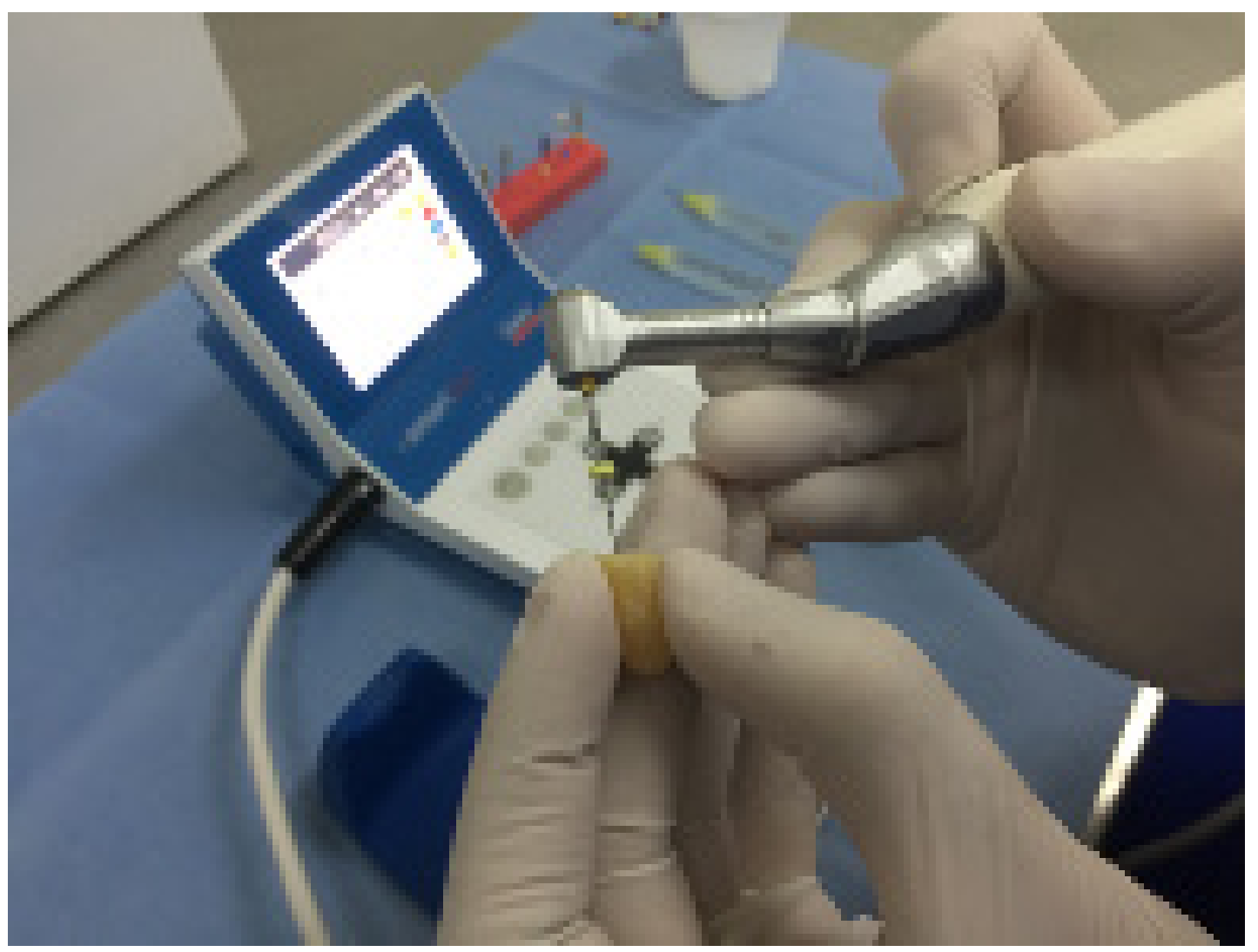

Figura 1. Proceso de preparación mecánica del conducto radicular con el sistema rotatorio Protaper Next (Dentsply/Maillefer)

Los dientes fueron divididos aleatoriamente en 3 grupos experimentales y 1 de control para ser obturados con conos de gutapercha X3 Protaper Next mediante técnica de cono único. Las muestras de los 3 grupos experimentales correspondían a: Grupo 1 (G1) Tubliseal - SybronEndo ( $n=20$ ), Grupo 2 (G2) Topseal - Denstply, Maillefer ( $n=20$ ), Grupo 3 (G3) BioRoot RCS - Septodont ( $n=20)$. Además, se contó con un grupo control positivo $(n=20)$ que corresponde a conductos obturados sin sellador (Figura 2). 


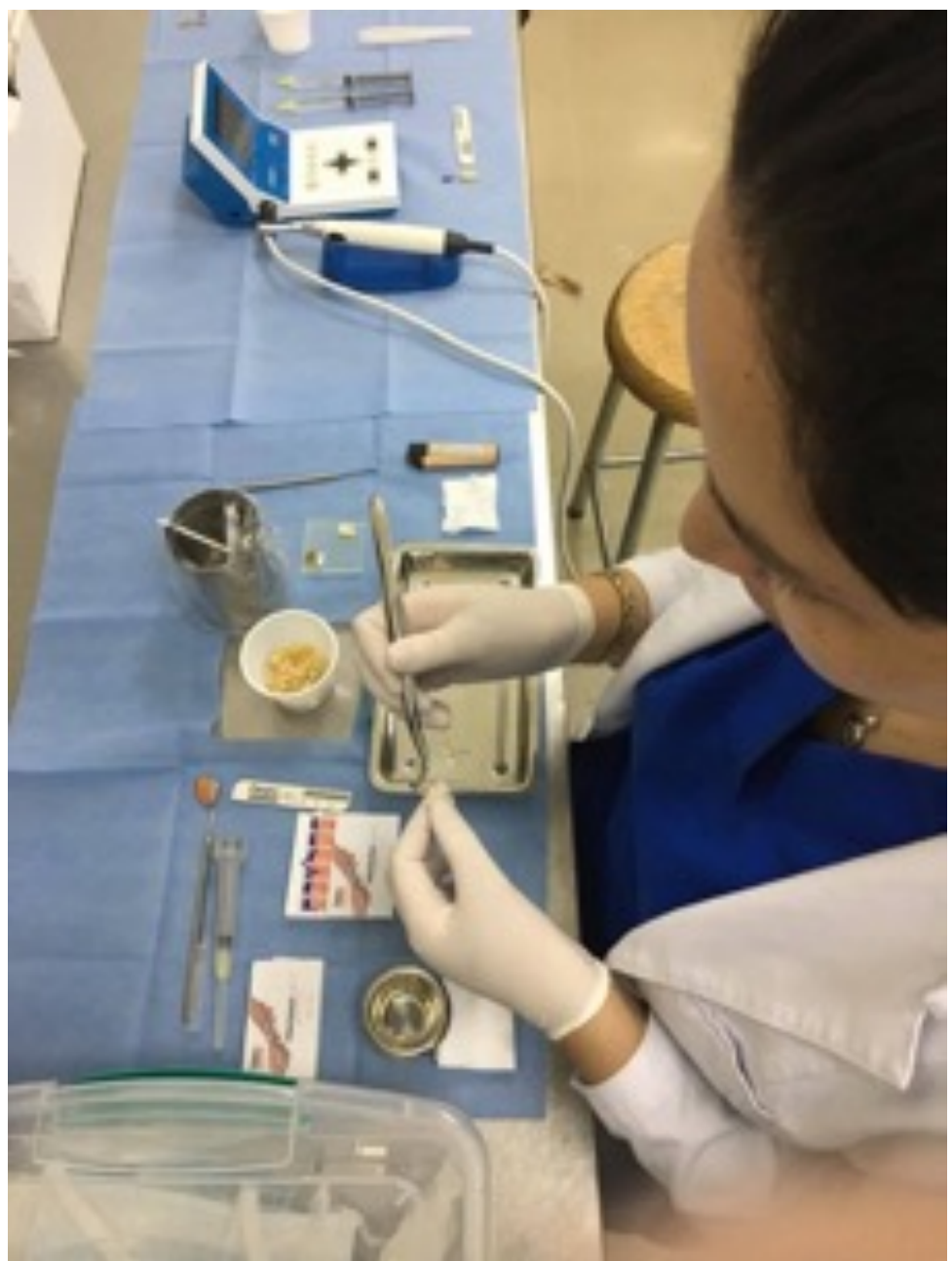

Figura 2. Proceso de Obturación con técnica de cono único

Las raíces fueron barnizadas con dos capas de esmalte de uñas (Opi) en su superficie radicular excepto en los $3 \mathrm{~mm}$ apicales. Una vez seco el esmalte, las muestras fueron sumergidas en recipientes con azul de metileno durante un periodo de 24 horas a temperatura ambiente (20+-1ํㅡ). Una vez terminado el proceso de tinción se lavó con agua corriente durante $15 \mathrm{mi}-$ nutos para eliminar los restos de azul de metileno y se retiró el esmalte de uñas transparente con bisturí (Denpher y Dochem) sin tocar el ápice.

Posteriormente las piezas se colocaron en recipientes para realizar la diafanización de acuerdo a la siguiente técnica (Figura 3 A y B):

1. Los dientes se sumergieron en Ácido Nítrico al $5 \%$ el cual se cambió cada 12 horas, a fin de lograr la descalcificación de las muestras, por 5 o 7 días según el caso, ya que algunas piezas se descalcifican más rápido que otras.

2. Luego se lavaron las muestras por 4 horas con agua corriente para retirar restos de Ácido Nítrico. Una vez limpias se secaron con secador de pelo (Gamma).

3. Todas las muestras fueron deshidratadas con metanol en concentraciones ascendentes según la siguiente secuencia: 
- Metanol al 50\%: Se sumergieron las muestras durante 2 horas.

- Metanol al 75\%: Se sumergieron las muestras durante 1 hora.

- Metanol al 90\%: se sumergieron las muestras durante 1 hora.

- Metanol al 99\%: se sumergieron las muestras durante 1 hora.

4. Una vez terminada la deshidratación se secaron con aire y se mantuvieron sumergidas en salicilato de metilo.
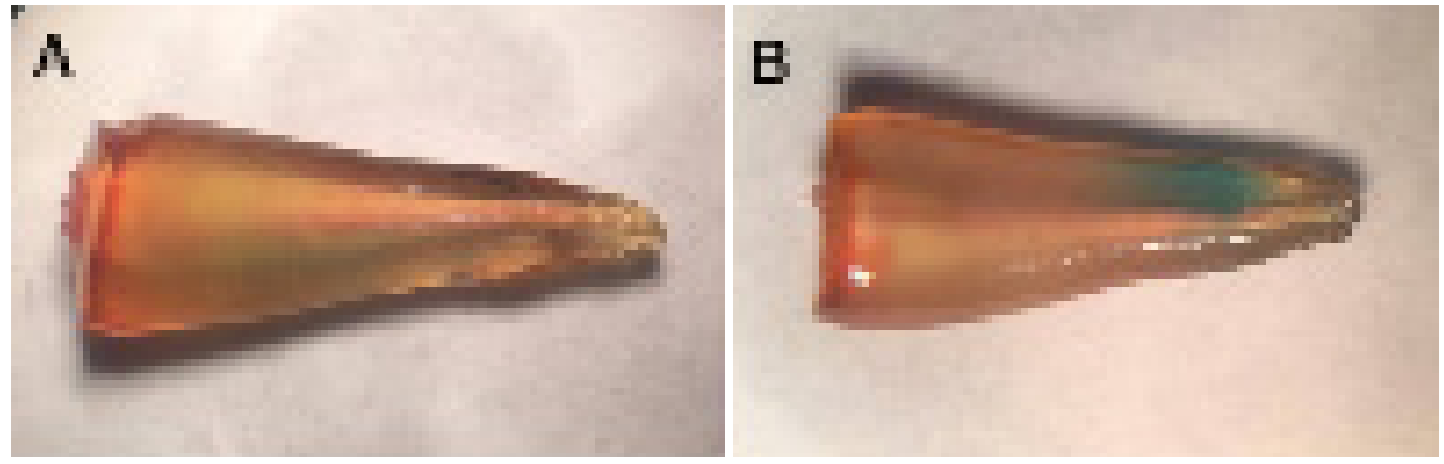

Figura 3. A y B: Raíces diafanizadas con ácido nítrico al $5 \%$ y etanol en grados crecientes.

Un único operador, que no conocía el cemento sellador empleado, observó las muestras con estereoscopio (Carl Zeiss) y midió la penetración lineal de azul de metileno en micrones ( $\mu \mathrm{m})$ mediante ocular micrométrico (Leitz Wetzlar) usando micrómetro de referencia (Spencer Lens Co.), desde el foramen. Las mediciones obtenidas de la penetración lineal del azul de metileno se registrarán en micrones ( $\mu \mathrm{m}$ )(Figura 4,5 y 6 ).
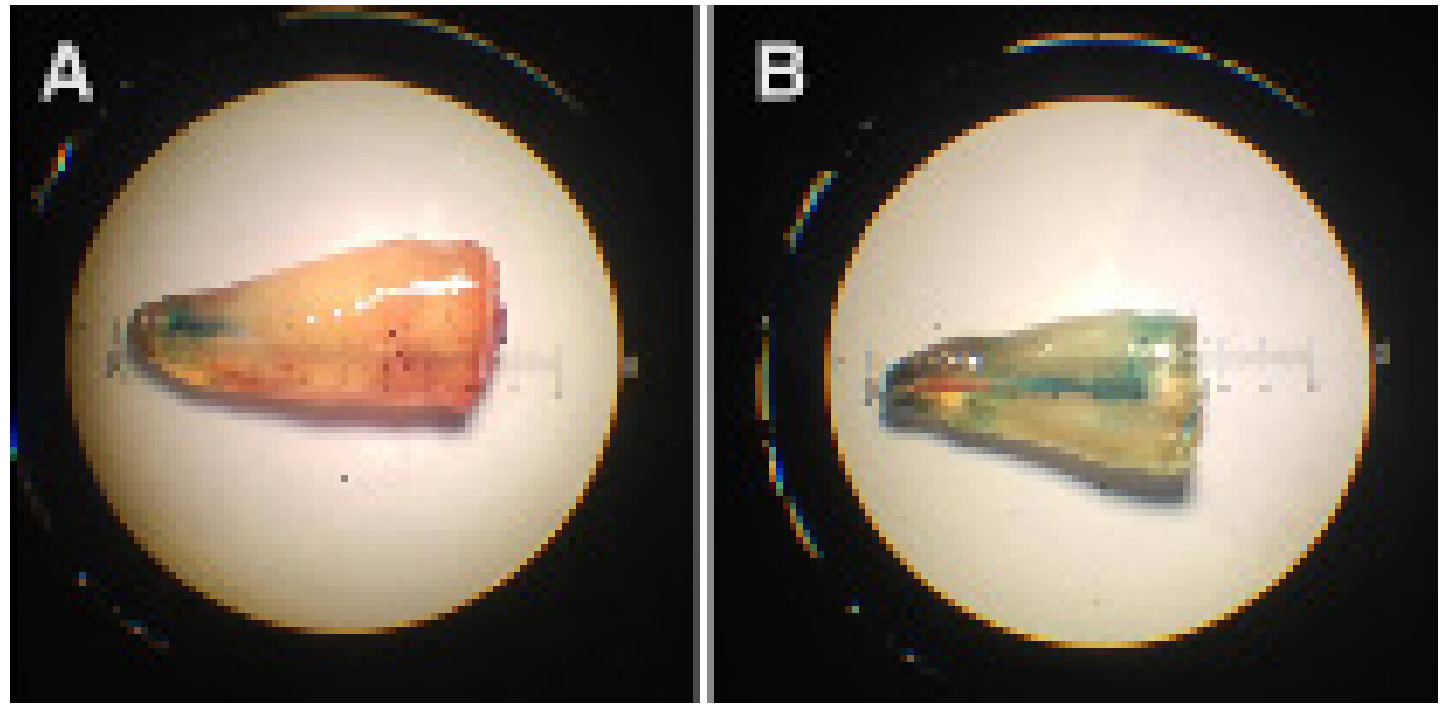

Figura 4. Raíces con sellador de conductos BioRoot. A: filtración únicamente del tercio apical, B: filtración a lo largo del conducto casi en su totalidad. 


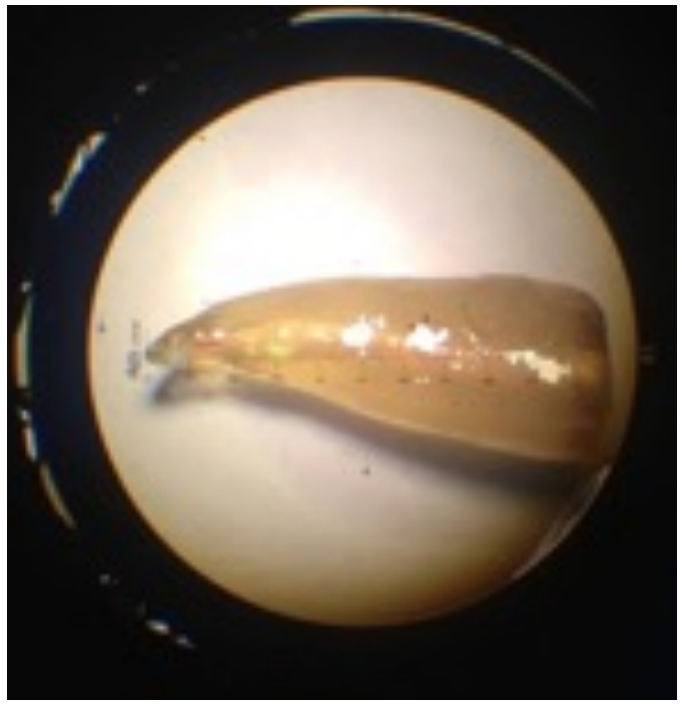

Figura 5. Raíz con sellador de conductos Tubliseal, no se observa filtración apical.
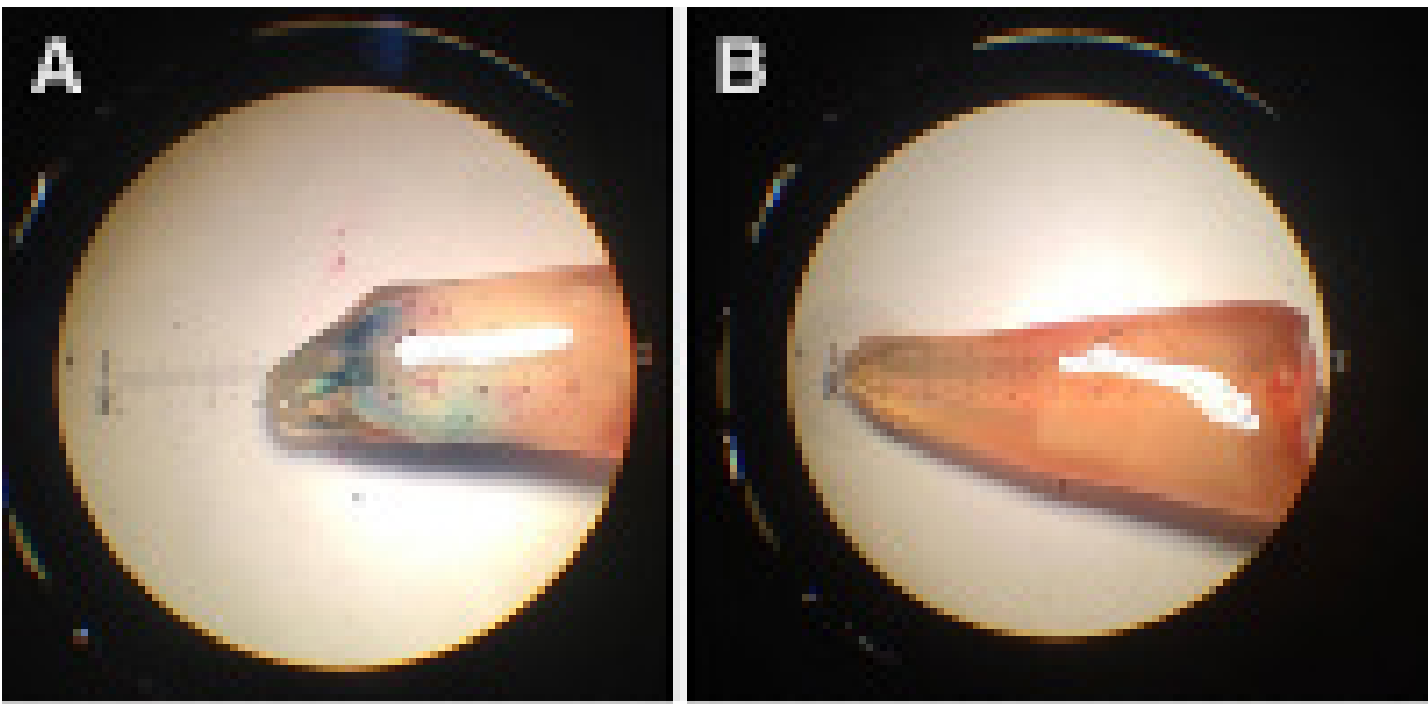

Figura 6. Raíces con sellador de conductos Topseal. A: evidencia filtración apical, B: no existe filtración apical.

Todas las muestras fueron eliminadas al término del estudio según el protocolo de manejo de residuos biológicos de la facultad de odontología de la Universidad de Concepción.

\section{Resultados}

Los datos obtenidos fueron tabulados en Microsoft ${ }^{\circledR}$ Office Excel $^{\circledR} 2015$ y analizados mediante el programa estadístico InfoStat ${ }^{\oplus} 2016$ con la prueba Kruskal Wallis con un nivel significancia del $5 \%$. Se utilizó esta prueba estadística no paramétrica porque los datos no mostraban una distribución normal.

La distribución anormal de los datos es reflejada por la gran dispersión de algunos valores en los grupos estudiados, esto se muestra en la Figura 7. 


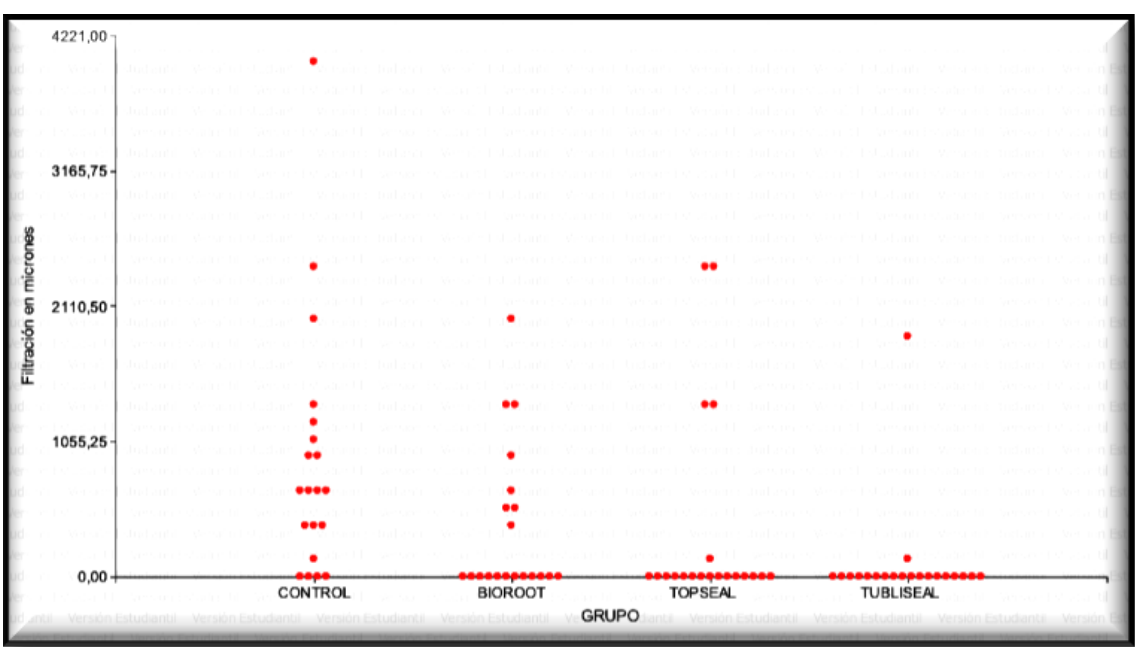

Figura 7. Dispersión de las medidas de filtración en los grupos estudiados. Los grupos experimentales BioRoot, Topseal y Tubliseal reflejan valores repetitvos, observando varios puntos en el inicio del eje ordinal del gráfico.

El grupo de control mostró la máxima penetración de la tinción, mostrando una mediana $670,00 \mu \mathrm{m}$ en comparación con los grupos experimentales que presentaron una mediana de $0,00 \mu \mathrm{m}$ (Tabla 1); la prueba evidencia que hay una diferencia estadísticamente significativa de 0,0002 entre los grupos.

\begin{tabular}{|c|c|c|c|c|c|}
\hline GRUPO & N & Medias & D.E. & Medianas & p \\
\hline BIOROOT & 20 & 388,60 & 592,77 & 0,00 & 0,0002 \\
\hline TOPSEAL & 20 & 381,90 & 805,50 & 0,00 & \\
\hline TUBLISEAL & 20 & 100,50 & 418,98 & 0,00 & \\
\hline CONTROL & 20 & 897,80 & 982,87 & 670,00 & \\
\hline
\end{tabular}

Tabla 1. Comparación entre grupos utilizando la prueba estadística Kruskal Wallis.

La diferencia estadísticamente significativa dada por la prueba estadística Kruskal Wallis establece la diferencia entre el grupo de control con los grupos experimentales que correponden a BioRoot RCS, Tubliseal - SybronEndo y Topseal - Denstply, Maillefer (Tabla 2)

\begin{tabular}{|c|c|c|}
\hline GRUPO & \multicolumn{2}{|l|}{ Ranks } \\
\hline TUBLISEAL & 28,75 & $\mathrm{~A}$ \\
\hline TOPSEAL & 36,20 & $\mathrm{~A}$ \\
\hline BIOROOT & 40,65 & $\mathrm{~A}$ \\
\hline CONTROL & 56,40 & $\mathrm{~B}$ \\
\hline
\end{tabular}

Tabla 2. Diferencia entre grupo control y grupos experimentales. 
En cuanto a la capacidad de sellado apical se observó que el grupo control presentó un valor mayor de microfiltración frente a los grupos experimentales con una media de 897,8 $\mu \mathrm{m}$. El Tubliseal es el cemento sellador que presentó una menor media de microfiltración $(100,5 \mu \mathrm{m})$ en relación a los grupos experimentales. Bioroot RCS y Topseal presentaron un comportamiento similar. Sin embargo, no hay diferencia estadísticamente significativa entre los cementos selladores de los grupos experimentales (Figura 8).

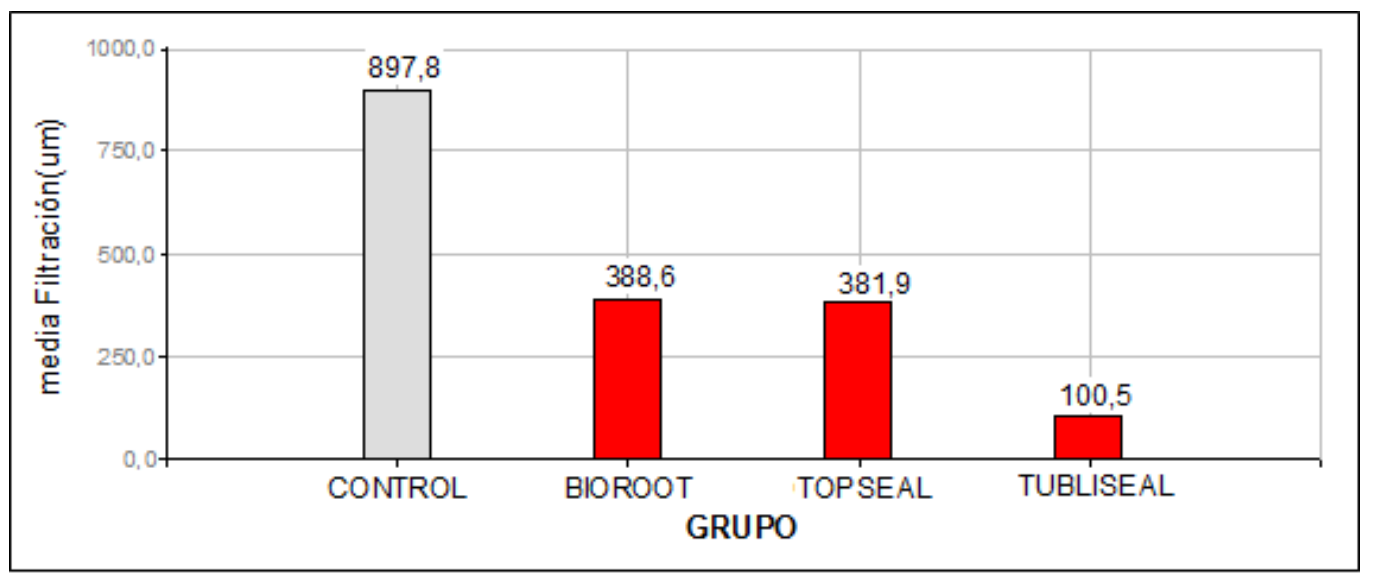

Figura 8. Media de microfiltración $(\mu \mathrm{m})$ de los grupos experimentales y grupo control

\section{Discusión}

El presente estudio evalúo la capacidad de sellado apical de diferentes selladores utilizando la técnica de obturación de cono único. La técnica de cono único en la actualidad es fundamentada por su simplicidad, puesto que se dispone de sistemas rotatorios con sus respectivos conos de gutapercha, además hay disminución de estrés en el diente, paciente y odontólogo ${ }^{20}$ En la literatura se evidencia estudios que comparan las técnicas de obturación cono único, compactación vertical caliente y compactación lateral dando como resultado que no existe diferencias significativas entre ellas. ${ }^{21}$

Al analizar los resultados de este estudio no se encontraron diferencias estadísticamente significativas entre los grupos experimentales, sin embargo se evidenció una diferencia de los grupos experimentales con el grupo control.

Consistente con nuestros resultados, Shetty $V$ et al. compararon la microfiltración apical usando la técnica de penetración de colorante, los selladores que compararon fueron Tubliseal, Sealapex y AH26. Ellos reportaron que Tubliseal mostró menor filtración en comparación a los otros selladores. ${ }^{22}$

Los grupos experimentales obturados con selladores Topseal y BioRoot presentaron valores similares de microfiltración, superiores a Tubliseal. 
Reszka P et al. menciona que las propiedades de sellado de BioRoot RCS combinadas con gutapercha son comparables a las de AHPlus, sin embargo su estudio realizado con microCT reveló un mayor volumen vacíos para BioRoot RCS que el sellador a base de resina, posiblemente debido al menor tiempo de trabajo y menor cantidad de AHPlus. ${ }^{8}$

BioRoot RCS mostró una mayor filtración que Tubliseal. Esto pudo originarse porque en la parte experimental no se consideró colocarlo en un medio con la humedad adecuada inmediato a la obturación. Siboni et al. menciona que los altos valores de solubilidad en agua destilada que presentó su grupo experimental obturado con BioRoot RCS se correlaciona con una alta liberación de $\mathrm{Ca} 2+$ y $\mathrm{OH}$-, que se disuelven dejando espacios vacíos. Cuando se sumerge en un fluido corporal simulado, los iones de calcio combinados con el fosfato forman una capa de Fosfato cálcico capaz de llenar los espacios vacíos(14). Por lo tanto la solubilidad y la porosidad de los materiales de silicato de tricálcico en agua destilada no predicen la estabilidad e integridad reales de dichos materiales in vivo. ${ }^{14}$

La evaluación de la capacidad de sellado es considerada un parámetro importante para evaluar la introducción al mercado de un nuevo sellador. Esta evaluación cada vez se ha visto desplazada por la falta de reproducibilidad en los estudios de microfiltración apical, esto se explica por las diferencias y complejidades anatómicas de los dientes investigados. ${ }^{19}$ Van der Sluis et al. demostró que existe una diferencia significativa en la filtración entre conductos ovalados y redondos, ${ }_{1}^{23}$ razón por la que nuestro estudio se pudo ver influenciado por la variación anatómica de los dientes utilizados, puesto que no se estandarizó la anatomía de los conductos previo al estudio, siendo necesario a futuro considerar esta característica.

\section{Conclusiones y recomendaciones}

En el presente estudio todos los grupos presentaron filtración apical, no obstante, el porcentaje de muestras que no filtraron en los grupos experimentales fue mucho mayor en relación a las muestras filtradas. Tubliseal mostró el mejor compartamiento de los selladores estudiados, aunque no existe una diferencia significativa con BioRoot RCS y Topseal, siendo estos útimos similares en su comportamiento.

BioRoot RCS presenta buenas propiedades de sellado, a en este estudio no se consideró simular el medio de humedad adecuado para su fraguado que a diferencia de otros selladores requiere un medio similar a los fluidos corporales con los que estaría en contacto cuando se usa in vivo.

El factor anatómico es una variable esencial a ser considerada en el diseño de estudios futuros, porque las variaciones en el tipo de diente, forma del conducto y calibre apical pueden influir directamente en los resutados de investigaciones de sellado apical. 


\section{Referencias Bibliograficas:}

1. Moradi S, Lomee M, Gharechahi M. Comparison of fluid filtraton and bacterial leakage techniques for evaluation of microleakage in endodontics. Dent Res J (Isfahan). 2015;12(2):109-14.

2. Lucian A, Bud M. The Use of Bioceramics in Endodontics - Literature Review. 2016;89(4):10-3.

3. Kim S, Kim S, Park J. Comparison of the Percentage of Voids in the Canal Filling of a Calcium Silicate-Based Sealer and Gutta Percha Cones Using Two Obturation Techniques. Mater. 2017;10(10).

4. Ahuja L. A Comparative Evaluation of Sealing Ability of New MTA Based Sealers with Conventional Resin Based Sealer: An In-vitro Study. J Clin Diagnostic Res. 2016;10(7):76-9.

5. Holland R, Murata SS. Apical Seal of Root Canals with Gutta-Percha Points with Calcium Hydroxide. Braz Dent J. 2004;15:26-9.

6. Prüllage R, Urban $K$, Schäfer E, Dammaschke T. Material Properties of a Tricalcium Silicate - containing, a Mineral Trioxide Aggregate - containing , and an Epoxy Resin - based Root Canal Sealer. J Endod. 2016;42(12):1784-8.

7. Patni PM, Chandak M, Jain P, Patni MJ. Stereomicroscopic Evaluation of Sealing Ability of Four Different Root Canal Sealers- An invitro Study. J Clin Diagnostic Res. 2016;10(8):379.

8. Reszka PB, Nowicka A, Lipski M, Dura WB, Dro A, Wo K. A Comparative Chemical Study of Calcium Silicate-Containing and Epoxy Resin-Based Root Canal Sealers. Biomed Res Int. 2016;

9. Baudry A, Richard G, Kellermann 0, Goldberg M. In vitro bioactivity of Bioroot TM RCS, via A4 mouse pulpal stem cells. Dent Mater [Internet]. 2015;31(11):1290-7. Available from: http://dx.doi.org/10.1016/j.dental.2015.08.163

10. Al-Haddad A, Aziz ZACA. Bioceramic-Based Root Canal Sealers: A Review. Int J Biomater. 2016;2016:1-11.

11. Khalil I, Naaman A, Camilleri J. Properties of Tricalcium Silicate Sealers. J Endod. 2016;42(10):1529-35.

12. Segatto A, Altmann P, Castelo V, Leitune B. Influence of Eugenol-based Sealers on Pushout Bond Strength of Fiber Post Luted with Resin Cement : Systematic Review and Meta-analysis. J Endod. 2015;41(9):1418-23.

13. Denstply/Maillefer. Topseal: Intrucciones de uso. 2008.

14. Siboni F, Taddei P, Zamparini F, Prati C, Gandolfi M. Properties of BioRoot RCS, a tricalcium silicate endodontic sealer modified with povidone and polycarboxylate. Int Endod J. 2017;0-3.

15. Swarup S, Rao A. Bioceramics in pediatric endodontics. 1st editio. Trivandrum: Lambert Academic Publishing; 2013. 53-68 p. 
16. Utneja S, Nawal RR, Talwar S, Verma M. Current perspectives of bio-ceramic technology in endodontics: calcium enriched mixture cement - review of its composition, properties and applications. Restor Dent Endod. 2015;40(1):1-13.

17. Best SM, Porter AE, Thian ES, Huang J. Bioceramics: Past, present and for the future. J Eur Ceram Soc. 2008;28(7):1319-27.

18. Badami V, Ahuja B. Review Article Biosmart Materials : Breaking New Ground in Dentistry. 2014;2014.

19. Viapiana R, Moinzadeh AT, Camilleri L, Wesselink PR, Filho MT. Porosity and sealing ability of root fillings with gutta-percha and BioRoot RCS or AH Plus sealers . Evaluation by three ex vivo methods. Int Endod J. 2016;49(8):774-82.

20. Sadr S, Golmoradizadeh A, Raoof M, Javad M. Microleakage of Single-Cone Gutta-Percha Obturation Technique in Combination with Different Types of Sealers. Iran Endod J. 2015;10(3):199-203.

21. Gordon MPJ, Love RM, Chandler NP. An evaluation of . 06 tapered gutta-percha cones for filling of . 06 taper prepared curved root canals. Int Endod J. 2005;38(3):87-96.

22. Shetty V, Hegde P, Chauhan Singh R, Rampratap Chaurasia V, Manmohan Sharma A, Taranath M. A Spectro Photometric Comparative Evaluation of Apical Sealing Ability of Three Different Sealers ; Calcium Hydroxide Based, Resin Based and Zinc Oxide Eugenol Based Sealers. J Int Oral Heal. 2015;7(2):25-7.

23. Van der Sluis $L$, Wu M, Wesselink P. An evaluation of the quality of root fillings in mandibular incisors and maxillary and mandibular canines using different methodologies. $J$ Dent. 2005;33:683-8.

Recibido:

Aceptado: 